\title{
TITLE
}

\section{Sex-specific responses of phenotypic diversity to environmental variation}

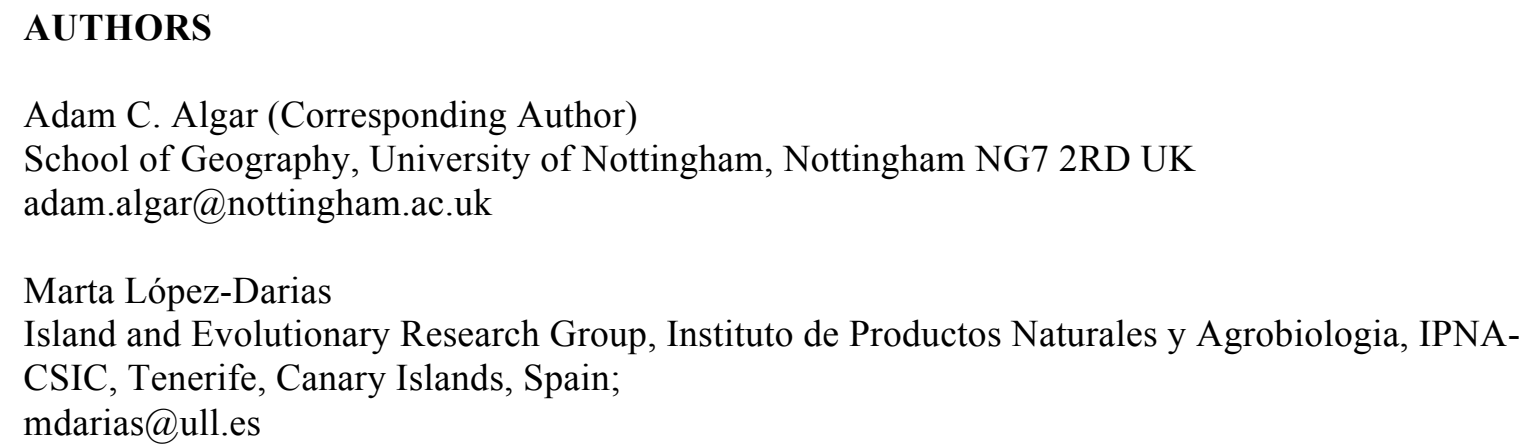

\section{KEYWORDS}

Convex hull, ecological radiation, ecomorphology, elevation gradients, environmental filtering, intraspecific variation, lizards, sexual selection, sexual size dimorphism, trait dispersion 


\section{ABSTRACT}

Identifying the factors generating ecomorphological diversity within species can provide a window into the nascent stages of ecological radiation. Sexual dimorphism is an obvious axis of intraspecific morphological diversity that could affect how environmental variation leads to ecological divergence among populations. In this paper we test for sex-specific responses in how environmental variation generates phenotypic diversity within species, using the generalist lizard Gallotia galloti on Tenerife (Canary Islands). We evaluate two hypotheses: the first proposes that different environments have different phenotypic optima, leading to shifts in the positions of populations in morphospace between environments; the second predicts that the strength of trait-filtering differs between environments, predicting changes in the volume of morphospace occupied by populations in different environments. We found that intraspecific morphological diversity, provided it is adaptive, arises from both shifts in populations' position in morphospace and differences in the strength of environmental filtering among environments, especially at high elevations. However, effects were found only in males; morphological diversity of females responded little to environmental variation. These results within G. galloti suggest natural selection is not the sole source of phenotypic diversity across environments, but rather that variation in the strength of, or response to, sexual selection may play an important role in generating morphological diversity in environmentally diverse settings. More generally, disparities in trait-environment relationships among males and females also suggest that ignoring sex differences in studies of trait dispersion and clustering may produce misleading inferences. 


\section{INTRODUCTION}

Evolutionary radiation can generate exceptional ecological diversity among species; however not all clades radiate ecologically (Schluter 2000, Losos and Mahler 2010, Blankers et al. 2012). Environmental features can both encourage evolutionary radiation, via ecological opportunity (Schluter 2000), or inhibit it, e.g. through insufficient area or heterogeneity (Ricklefs and Lovette 1999, Losos and Schluter 2000, Algaer and Mahler 2015). Understanding how, and when, environmental variation influences ecological diversification is thus a key question for evolutionary biologists and ecologists (Blankers et al. 2012, Kaliontzopoulou et al. 2014).

The relationship between a species' environment and its morphological variability has received substantial attention in a wide variety of lizard groups. For example, limb length is known to be adaptive for improved locomotion on particular substrates, such as narrow versus broad perches (Anolis; Losos et al. 2000), open versus closed habitats (Niveoscincus; Melville and Swain 2000) or terrestrial versus arboreal habitats (chameleons; Bickel and Losos 2002). Other traits, such as head characteristics (Huyghe et al. 2007, Measey et al. 2009) and colour pattern (Forsman and Shine 1995) are also known to differ among environments in lizards. At broader scales, body size shows considerable variability along elevation and latitudinal clines (Ashton and Feldman 2003, PincheiraDonoso et al. 2008, Muñoz et al. 2014a). Other traits, such as physiological tolerance, visual system, and behavior also vary with environmental conditions in lizards (Leal and Fleishman 2002, Ord et al. 2010, Johnson et al. 2010, Muñoz et al. 2014b).

Studies of morphological variation within clades and communities have traditionally focused on mean differences among species, and thus attempt to reconstruct the drivers of ecological diversification after the fact. An alternative approach is to examine how morphological variation is generated among populations that have not (yet) undergone speciation, providing insight into the nascent stages of ecological diversification and adaptive radiation (Thorpe and Baez 1987, Thorpe et al. 2010). More generally, identifying the factors driving intraspecific morphological variation can also provide 
insight into the factors driving ecological function and dynamics within communities (Bolnick et al. 2011, Violle et al. 2012).

As populations diversify, they can fill ecological space in new ways. Specifically, environments may differ in their ecological optima, leading to directional selection and shifts in a population's position within ecological space. For example, Caribbean Anolis lizards have repeatedly evolved toward different phenotypic optima depending on their microhabitat use (Mahler et al. 2013). Environments may also differ in the range of ecological variation they can support, i.e. in the strength of the environmental filter they impose (Weiher et al. 1998, Cornwell et al. 2006, Violle et al. 2012), constraining the volume of ecological space occupied by a species, or population (Weiher et al. 1998, Cornwell et al. 2006, Algar et al. 2011, Violle et al. 2012). These two options are not mutually exclusive and could act in concert or opposition to influence how populations fill ecological space across heterogeneous environments.

Sexual dimorphism is a fundamental axis of morphological variation in animals (e.g. Fairbairn et al. 2007) and may contribute substantially to ecological and lineage diversification in a number of ways. Sexual selection, which can produce sexual size dimorphism via male-male competition or fecundity selection in females (Cox et al. 2003), has been identified as a driver of speciation (Panhuis et al. 2001, Hudson and Price 2014). However, sexual dimorphism may also inhibit diversification if males and females of dimorphic species use a wide range of resources (Bolnick and Doebeli 2003, Ritchie 2007). Sexual dimorphism may also reflect both natural and sexual selection: Lopez-Darias et al. (2014) showed, in Gallotia lizards in the Canary Islands, that the same traits (head size and bite force) may be under sexual selection for one sex (males) and natural selection for the other (females). Regardless of whether dimorphism arose from sexual or natural selection, morphological differences between males and females can translate into ecological differences (Schoener 1967, Shine 1989, Butler et al. 2000, Butler and Losos 2002, Bolnick et al. 2011), raising the possibility that males and females may respond differentially to environmental variation. For example, Butler \& Losos (2002) found that Anolis ecomorphs in different microhabitats also varied in their degree of sexual 
dimorphism. Similarly, habitat $\times$ sexual dimorphism interactions have been identified within lacertid lizards, where populations diverge sexually to varying extents in traits, like limb length, related to habitat use (Kaliontzopoulou et al. 2010). The degree of sexual size dimorphism can also vary across broad scale climatic gradients (Fitch 1981, Stillwell and Fox 2009, Laiolo et al. 2013), though such patterns are variable across taxa (Blanckenhorn et al. 2006). Rensch's rule states that sexual size dimorphism increases with body size; in species with larger males, this is due to greater variation in males than females (Abouheif and Fairbairn 1997). Though Rensch's Rule applies inconsistently within species (Blanckenhorn et al. 2006), it suggests that, in species with larger males, differences in sexual dimorphism among environments will arise from variation in males, rather than females.

In this paper we test whether differences in how populations fill morphological space (morphospace) in different environments arise from differences in the phenotypic optima among environments (optimum-shift hypothesis), or in the strength of environmental filtering (environmental filter-strength hypothesis), or a combination of both. The optimum-shift hypothesis predicts that a population's centroid in morphospace will be further from the species' (all populations pooled) centroid than expected based on a null model of randomly assembled populations. The environmental filter-strength hypothesis predicts that the volume of morphospace occupied by a population (i.e. its convex hull; Cornwell et al. 2006) will be smaller than predicted based on the same null model, i.e. traits will be clustered. We also evaluate whether the response of morphospace filling to environmental variation differs between males and females. We test these hypotheses for Gallotia galloti, a widespread, generalist lizard on the environmentally diverse island of Tenerife (Canary Islands).

\section{METHODS}

\section{Study system}

Tenerife, the largest island in the Canary Islands is environmentally very diverse. Covering over $2,000 \mathrm{~km}^{2}$ and rising to more than $3,700 \mathrm{~m}$ above sea level, it hosts a wide range of habitats, including sub-desert coastal scrub, thermophilous, pine and laurel forest, and high elevation scrub/alpine ecosystems (Fernández-Palacios and Whittaker 2008, Zobel et al. 2011). These habitats arise in large 
part from the influence of elevation and orientation with respect to the humid northeast trade winds (Fernández-Palacios and Nicolás 1995, Fernández-Palacios and Whittaker 2008). Although human habitat modification, primarily through agriculture and tourism-related development, has greatly reduced the extent of most natural habitats, it has also generated agricultural and urban habitats at the regional scale (Fernández-Palacios and Whittaker 2008).

Although Tenerife (and the Canaries more generally) harbours several stunning evolutionary radiations, especially of plants and arthropods (e.g. Arnedo et al. 2001, Arechavaleta et al. 2009, Vitales et al. 2014), the lizard fauna has not radiated extensively within islands (Cox et al. 2010). Just four native lizard species inhabit Tenerife: one endemic gecko, one endemic skink, and two extant lacertid species of the endemic Canarian genus, Gallotia. Of these, G. galloti is by far the most widespread and abundant. Its only extant putative lizard competitor, G. intermedia, is extremely geographically restricted, limited to a small area of cliff habitat. Thus, despite Tenerife's substantial environmental diversity, its herpetofauna is dominated by a single, widespread species.

G. galloti is a medium sized, omnivorous lizard (Fig. 1), restricted to Tenerife and La Palma and is one of the dominant and most conspicuous native vertebrates on both islands. G. galloti's ancestor likely colonized Tenerife's proto-islands 9-10 mya (Cox et al. 2010) and it currently occupies every major habitat and geographical region in Tenerife, though its abundance in the northern tip of the island, Anaga, seems to be markedly lower than elsewhere (Thorpe and Baez 1987). It and its congeners are sexually dimorphic, especially in body size (males are larger), but also, for most species, in body shape, limb length and head size (Thorpe and Baez 1987, Molina-Borja et al. 1997, 2010, Herrel et al. 1999, Molina-Borja 2003, Lopez-Darias et al. 2014).

In addition to substantial sexual dimorphism, G. galloti also exhibits extensive morphological variation through space. Thorpe and Baez (1987) surveyed 18 populations of G. galloti across Tenerife and identified considerable geographical variation in body and head size, scalation and colour. However, geographical concordance across sexes and traits varied considerably. For example, 
body size showed very different patterns in males and females, while head size varied similarly between sexes (though males had larger heads). Traditionally, phenotypic differences have led to the delineation of two subspecies (or races) on the island (G. galloti galloti and G. galloti eisentrauti) that differ in colouration (Thorpe and Baez 1987, Molina-Borja et al. 1997). Gallotia g. eisentrauti also tends to be larger (Molina-Borja et al. 1997), though this difference is less clear when variation in $G$. g. gallotia due to contact zones and elevation is incorporated in the comparison (Thorpe and Baez 1987).

\section{Quantifying environmental variation}

We identified areas of similar environmental conditions on Tenerife, following the approach used by Hortal and Lobo (2005) and Hortal et al. (2007). Firstly, because climatic variables tend to be correlated through space, we performed a principal component analysis on climate and topographic variables sampled at a $500 \mathrm{~m}$ resolution across. We included the following variables: elevation, aspect, and mean annual, maximum and minimum temperature and annual, monthly minimum and maximum precipitation (Fig. A1; raw data from Hortal et al. (2006), shared by J.M. Lobo). For aspect, we computed 'northness' [northness $=$ cosine $($ aspect $)]$ and 'eastness' [eastness $=$ sine $($ aspect $)]$, where aspect is measured in radians. We extracted the first three principal components for the subsequent clustering analysis. Environmental PCs 1-3 accounted for $88 \%$ of the total variance (Table A1) and co-varied with temperature and elevation (envPC1), aridity and northness (envPC2) and eastness (envPC3), respectively. These captured the three major axes of environmental and ecosystem variation on Tenerife (Fig. A2).

We used k-means clustering of environmental PCs 1-3 to delineate environmental zones on Tenerife. We used $k=4$ as this captured $70 \%$ of the environmental variation on Tenerife with a tractable number of environmental zones to encompass variation in our model organism (Fig. 2). Environment A is found at low southern elevations characterized by high temperatures and low rainfall. Environment B is found at mid elevations, with southern exposure, cooler temperatures and more rainfall than Environment A. Environment C represents the north of the island at low to mid 
elevations, and has northerly slopes with much higher rainfall and humidity due to the influence of the trade-winds. Environment D is found at the highest elevations on the Teide volcano, and is characterized by extreme temperatures, especially cold, and widely seasonal precipitation patterns (including snow).

\section{Lizard sampling}

We sampled lizards in August 2011 at 20 sites across Tenerife. Sites were representative of the major environmental zones (Fig. 2). At each location, we trapped lizards for one to four consecutive days using pitfall traps baited with tomato or banana. After we measured them, lizards were released precisely where they were caught. Captured lizards were temporarily marked with a marker pen on the abdomen to avoid re-measuring the same individual. At each site, we aimed to trap 10-12 lizards of each sex (identified by appearance and/or everting hemipenes or checking the abdomen for eggs). Individuals that could not be reliably sexed (e.g. females versus juvenile males) were excluded. At some sites, the target sample size could not be obtained, while at others, high abundance meant more lizards could be measured (Table A2).

\section{Quantifying morphological variation}

We measured 14 traits relating to body size (snout-vent length; SVL), leg and toe length, and head length, height and width (Table 1; Fig. A3). MLD performed all measurements to eliminate measurer effects. To account for the effect of body size, we regressed the logarithm of each trait on log SVL and used the residuals in subsequent analyses (e.g. Pinto et al. 2008). Next, we identified key axes of morphological variation using a principal component analysis on these residuals (Table A3). We retained the first three principal components, which accounted for $73 \%$ of the total variance (Table $\mathrm{A} 3 ; \mathrm{PC} 1=54.3 \%, \mathrm{PC} 2=11.4 \%, \mathrm{PC} 3=7.2 \%)$. We used a varimax rotation to clarify the loading structure and increase interpretability. After rotation, PCs 1-3 loaded heavily on head size, toe length, and leg length, respectively (Table 1).

\section{Testing for trait and sexual dimorphism variation across environments}


We used linear mixed models to test for differences in trait means and sexual dimorphism across environments. For each trait (SVL; head size: PC1; toe length: PC2; and leg length: PC3), we first tested for variation in sexual dimorphism across environments using a model with a sex-environment interaction. We included environment and sex, and their interaction, as fixed effects and site as a random effect using the nlme package (Pinheiro et al. 2014) in R. If the environment by sex interaction was not significant ( $\alpha=0.05$, Wald's test), we removed it from the model. We compared models assuming equal variances with models that accounted for differing variances among environments using $\mathrm{AIC}_{\mathrm{c}}$.

\section{Testing the optimum-shift and environmental filter-strength hypotheses}

We used three morphological axes for the morphospace analysis: SVL (log-transformed), head size (PC1), and leg length (PC3). We omitted toe length (PC2) because we found no differences between environments or sexes in this trait (Table 2). We standardized each trait (logSVL, PC1 and PC3) to have a zero mean and unit variance before computing convex hulls. Estimates of convex hull volume are sensitive to sample size; larger samples are more likely to contain extreme values. To account for unequal sampling across environments, we randomly subsampled 20 males and 20 females from each environment and used these subsets to compute convex hull volumes. We repeated this sub-sampling 100 times and used the mean in subsequent analyses.

We measured the volume of morphospace occupied by lizards from each environment and all environments together using convex hull analysis (Cornwell et al. 2006), where morphospace was defined by three axes: body size, head size and limb length. Our measure of volume is equivalent to the functional richness of a species assemblage (Villéger et al. 2008). Next, we measured the morphological dissimilarity of lizard populations among the four environments, following Villéger et al (2011). The morphological dissimilarity is the percentage of morphospace volume that is occupied uniquely by, in this case, lizards from a single environment. We computed the total morphological dissimilarity among all environments, as well as all six pairwise combinations. We used the 'hypervolume' package (Blonder et al. 2014; Blonder 2015) in R v.3.2.1 (R Core Team 2015) to 
compute convex hulls for each environment and their unions. We repeated this analysis for each trait individually, using trait ranges instead of convex hulls. All analyses were carried out with both sexes pooled and for males and females separately.

To test the environmental filter-strength hypotheses, we compared the convex hull volume for lizards from each environment to the volume expected from a null model. For the optimum-shift hypothesis, we calculated the distance from each population's morphospace centroid to the centroid of all populations pooled and compared this to a null expectation. The null expectation was created for each environment by randomly selecting 40 individuals (20 males and 20 females) without replacement from the entire pool of individuals. We repeated this 1000 times to compute P-values and their $95 \%$ confidence intervals following Ruxton and Neuhäuser (2013), with the observed value included in both the numerator and denominator of P-value calculations. All tests were two-tailed.

\section{RESULTS}

\section{Testing for trait and sexual dimorphism variation across environments}

We found considerable sexual size dimorphism in Gallotia galloti, with males achieving much greater SVL than females (Figs. 3 and 4). However, we also found a significant interaction between sex and environment in our linear mixed effects model (Table $2 ; \mathrm{F}_{3,328}=7.69, \mathrm{P}<0.001$ ) indicating that the degree of sexual size dimorphism varied among environments. No other traits showed a significant sex $\times$ environment interaction (Table 2). Models assuming equal variances among environments had lower $\mathrm{AIC}_{\mathrm{c}}$ values than models that allowed variances to vary for all traits except head size (PC1 $\left.\Delta \mathrm{AIC}_{\mathrm{c}}=5.6\right)$. Thus for this trait, we present results from models allowing unequal variances among environments. Results were nearly identical (and there were no changes in significance) regardless of which models were used.

Once the sex $\times$ environment interaction was removed, we found significant sexual dimorphism in head size (PC1) and leg length (PC3), but not toe length (PC2; Table 2). Males tended to have larger heads and longer legs, relative to body size, than females. Head size (PC1) and leg length (PC3) also 
varied across environments, independently of sex, $(\mathrm{P}=<0.02$ for both; Table 2$)$. The trend was for greater relative male head size and longer relative leg lengths at high elevations (Environment D; Fig. $3)$.

\section{Environmental variation and morphological dissimilarity}

Morphological dissimilarity, i.e. the percentage of morphospace uniquely occupied by lizards from a single environment, was $36 \%$ (Fig. 5), but this was not significant based on our null model ( $\mathrm{P}=0.15$, $\left.\mathrm{CI}_{95}=0.13-0.18\right)$. Individual contributions of environments to this value ranged from $3 \%$ to $17 \%$ (Table A4), but only Environment C's unique volume differed significantly from the null expectation, though the $95 \%$ confidence interval still overlapped $0.05\left(\mathrm{P}=0.041, \mathrm{CI}_{95}=0.029-0.053\right)$. Pairwise dissimilarity (i.e. morphological turnover) among environments ranged from $51 \%-74 \%$, but only comparisons involving the high elevation Environment D were significant (Table A5).

Partitioning the data by sex revealed different patterns among males and females. Males underwent more morphological turnover between environments than females ( $61 \%$ versus $42 \%)$. Moreover, for males, this morphological turnover was significant $\left(\mathrm{P}=0.023, \mathrm{CI}_{95}=0.014-0.032\right)$ while for females it was not $\left(\mathrm{P}=0.43, \mathrm{CI}_{95}=0.39-0.45\right)$. However, uniquely occupied volumes were not significant for any environment for either males or females, though P-values were low for Environment C (Table A4), suggesting no single environment drives morphological dissimilarity. Pairwise morphological dissimilarity for males was higher than for females. For the former, all environments differed significantly from Environment D, while for females only Environments B (mid elevation) and D (high elevation) differed significantly (Table A5).

\section{Individual trait dissimilarity among environments}

When sexes were pooled, individual trait dissimilarity was $20 \%$ for body size (log SVL), $14 \%$ for head size (PC1) and 11\% for leg length (PC3), though only body size dissimilarity was significant (P $\left.=0.004, \mathrm{CI}_{95}=0.000088-0.0008\right)$. Males and females both showed greater dissimilarity for body size 
than other traits, with males having greater dissimilarity than females for all traits. However, only male body size dissimilarity was significant (Table A6; $\mathrm{P}=0.002, \mathrm{CI}_{95}=0.001-0.0048$ ).

With sexes pooled, the only significant pairwise comparisons were for body size turnover between environments B versus D, C versus D, and B versus C (Table A7). However, for males, several significant pairwise differences were found for both body size (Table A7; B versus D, and C versus D) and head size (Table 6; Environment D differed from all others). For females, body size turnover was not significant and only a single comparison was significant for leg length (Table A7; Environment B versus D).

\section{Testing the environmental filter-strength and optimum-shift hypotheses.}

When both sexes were considered together, populations at mid and high elevations (Environments B and D in Fig. 2) occupied less morphospace than lower elevation environments (Fig. 5). This clustering was strongest and significant at the highest elevations (Environment $\mathrm{D} ; \mathrm{P}=0.011, \mathrm{CI}_{95}=$ 0.0045-0.017), but weak at mid elevations (Environment B; Fig. 5; $\mathrm{P}=0.058, \mathrm{CI}_{95}=0.043-0.072$ ). Neither the low elevation northern or southern environments (Environments A and C) displayed significant clustering $(\mathrm{P}>0.75$ for both). Males occupied significantly less morphospace, i.e. were more clustered, at high elevations (Environment D) than expected due to chance (Fig. 5; $\mathrm{P}=0.033$, $\left.\mathrm{CI}_{95}=0.022-0.044\right)$, but were not clustered in any other environment ( $\mathrm{P}>0.30$ in all cases). Although patterns of females appeared qualitatively similar to those of males, they exhibited less variation and displayed no significant clustering in any environment (Fig. 5; P > 0.12 in all environments).

Pooling both sexes, the mid and high elevation environments ( $\mathrm{B}$ and $\mathrm{D}$, respectively) showed greater centroid displacement than the two low elevation environments. Of the former set, Environment D was more severely displaced (Fig $5 ; \mathrm{P}=0.001, \mathrm{CI}_{95}=0.001-0.0029$ ) relative to the null expectation than the lower elevation Environment B (Fig. 5; $\mathrm{P}=0.006, \mathrm{CI}_{95}=0.0012-0.011$ ). Once again, the strong departure of the high elevation Environment D was driven by male variation (Fig. 5). Males from Environment D were much further from the pooled morphospace centroid than expected from 
our null model $\left(\mathrm{P}=0.002, \mathrm{CI}_{95}=0.001-0.0048\right)$. Females show no such displacement at the highest elevations, but did for the mid-elevation Environment B (Fig. 5; $\mathrm{P}=0.002, \mathrm{CI}_{95}=0.001-0.0048$ )

\section{DISCUSSION}

Identifying how environmental factors generate ecomorphological variation within species provides a window into the nascent stages of ecological radiation. On Tenerife, just over one third of the morphospace occupied by the lizard Gallotia galloti (Lacertidae) was uniquely occupied by lizards from a single environment, indicating that environmental differences are accompanied by morphological turnover (sensu Villéger et al. 2011) among populations. We tested two pathways by which environmental variation may have generated these differences between populations within a species, and thus enhance morphological diversity. The optimum-shift hypothesis proposes that the phenotypic optimum differs between environments, leading to divergence in ecomorphological traits between populations in different environments (Mahler et al. 2013). The environmental filter-strength hypothesis suggests that the strength of environmental filtering varies across environments, leading to differential trait-clustering (Weiher et al. 1998, Algar et al. 2011, Swenson et al. 2012). Thus, under an optimum-shift model, environment-specific selection pressures act primarily on phenotypic position in morphospace, while under an environmental filter-strength model, they act primarily on phenotypic variance.

We found that populations' morphospace volumes and centroid displacements (the distance of a population's centroid in morphospace from the overall centroid) varied among environments, and that this variation was elevationally structured. At low elevations (Environments A and C in Figure 3), neither morphospace volume nor centroid displacement differed from that expected based on random sampling from the total population. However, at mid to high elevations (Environments B and D), populations' morphospace volumes were less and centroid displacements were greater than the null expectation, suggesting an increase in trait clustering at high elevations and a shift in trait optimums. 
However, the intraspecific morphological diversity observed in G. galloti across environments arose from environmentally structured morphological variation in males, but not in females. Variation in male lizards across environments primarily occurred along the body size axis, with weak variation in leg length and head size. Males tended to be smaller at high elevation, a common, but not ubiquitous, pattern within lizards (Ashton and Feldman 2003, Pincheira-Donoso and Meiri 2013, Muñoz et al. 2014a). In contrast, we found that female patterns of morphospace occupation did not differ from random expectations, with the single exception of centroid displacement in Environment B. Thorpe and Baez (1987) suggested that geographical patterns of male and female body size on Tenerife were incongruent. Our findings suggest that these differences - and concomitant changes in sexual dimorphism — arise, at least in part, from reduced morphological differentiation of females among environments, compared to males. This pattern is consistent with the phenomenological Rensch's rule, which suggests greater size variation among males than females (Blanckenhorn et al. 2006).

Although our results are consistent with both the optimum-shift and environmental-filter strength hypotheses, the discrepancy between male and female variation reveals a more complex story. The larger shifts in morphospace position of $G$. galloti males, relative to females, suggest that changes are not simply a function of natural selection for different phenotypic optima, or reduced phenotypic variance, across environments. The large body and head size of G. galloti males is thought to reflect male - male competition (Molina-Borja et al. 1997, Herrel et al. 1999, Huyghe et al. 2005, LopezDarias et al. 2014), while female head size across Gallotia varies with prey size and hardness (LopezDarias et al. 2014). Based on a link between head size, bite force and diet in females, but a decoupling of bite force and diet in males, Lopez-Darias et al. (2014) concluded that male Gallotia morphology is driven by sexual selection and female morphology by natural selection. If differences in the direction of natural selection were solely responsible for shifts in morphospace among populations, then females should have responded at least as strongly, if not more so, than males. Instead, the stronger response of males suggests a possible gradient in the strength of sexual selection across environments. For example, the strength of (or responses to) sexual selection may be stronger in warm, aseasonal environments on Tenerife than in harsh, high elevation locales, where the need to cool and warm 
quickly during substantial daily and annual temperature fluxes (Ashton and Feldman 2003, Sears and Angilletta 2004), may be more important. Such a scenario would suggest that, at low elevations, natural and sexual selection act in concert (e.g. Irschick et al. 2008), but may oppose each other at high elevations. Suggestively, relative head size of males tended to increase at high elevations, suggesting that male head size may still be under selection despite smaller body sizes.

The above interpretation relies on trait values reflecting adaptive processes in different environments. However, it is entirely possible that the morphological variation observed here along environmental gradients could be non-adaptive (Angilletta et al. 2004, Sears and Angilletta 2004). Lower resource availability and/or activity time at high elevations could reduce growth rates and body size, especially if juvenile mortality is high (Sears and Angilletta 2004). Evidence in Anolis lizards suggests that male growth can be affected by resource constraints more than females, reducing sexual size dimorphism in resource-limited environments (Cox and Calsbeek 2010). Thus, the environmentally structured morphological variation in male G. galloti lizards may reflect plastic responses that prevent males from reaching large body sizes, rather than variation in the strength of selection (natural or sexual) through environmental and geographic space.

In addition to potential shifts in the trait optimum among environments, we also found evidence that there is stronger environmental filtering, leading to greater trait clustering, at the highest elevations. Strong filtering in this environment is consistent with the hypothesis that harsh environments (such as those at high elevations or latitudes) limit the range of traits or strategies that can occur (Weiher and Keddy 1995, Weiher et al. 1998, Cornwell et al. 2006, Swenson et al. 2012). High elevation environments (e.g. summit scrub) in Tenerife are characterized by cool average temperatures, low productivity and marked temperature variability, with frost and snow common during winter months (Zobel et al. 2011). Recent volcanic activity may also have contributed to environmental harshness and has been hypothesized to reduce lizard body sizes (Muñoz and Hewlett 2011). The environmental filter operating at high elevations seems to especially exclude large males (Figs. $3 \& 5$ ). Such an asymmetric environmental filter could also contribute to a shift in a population's centroid, even if the 
actual trait optimum does not shift. Under this scenario, we would expect the distribution of male body sizes at high elevations to appear truncated, or strongly left-skewed, because of the absence of large males. However, we see no obvious skew in the male body size distributions at high elevations (Figure A4), suggesting that the observed centroid shift is not solely a function of asymmetric filtering, and that both shifts and filtering are operating at higher elevations. Variation in filtering likely arises from the same processes hypothesized above: selection against large males and/or plastic responses due to restricted activity times, lower temperatures, and lower resource availability.

Intraspecific morphological diversification in response to spatially varying environmental pressures, i.e. ecological diversification, may presage genetic isolation and speciation (Thorpe et al. 2010, Muñoz et al. 2013). G. galloti on the main island of Tenerife, i.e. excluding islets, has traditionally been divided into two subspecies, G. g. galloti and G. g. eisentrauti. Roughly speaking, the former inhabits southern environments on Tenerife, while the latter inhabits the north, including our Environment C (Thorpe and Baez 1987). However, our results suggest that lizards from Environment C were not morphologically exceptional, thus morphological diversification pressures in G. galloti have likely not driven sub-species formation, which instead may reflect historical isolation on precursor islands (Thorpe et al. 1996) or differentiation of other traits not measured here, such as colouration (Molina-Borja et al. 1997, 2006). Lizards from Environment C, especially males, did tend to be large (Fig. 3, also see Molina-Borja et al. 1997), but so did individuals of G. g. galloti in Environment A. Environment C also contributed the most to overall morphological dissimilarity, probably because it had the most variable body sizes (Fig. 3), but did not display significant clustering or centroid displacement. Instead, the primary axis of morphological differentiation of G. galloti on Tenerife was between the sexes, and secondarily along the island's elevation gradient. Sexual dimorphism can inhibit lineage diversification because dimorphic males and females may use a wider range of ecological resources (Bolnick and Doebeli 2003). Thus the substantial sexual dimorphism of G. galloti could have inhibited speciation within Tenerife, though a small island size (Losos and Schluter 2000) and short time for speciation have also likely played a role. 
Identifying how environmental variation generates morphological diversity and differentiation among populations can provide a window into the embryonic stages of ecological diversification and, possibly, ecological speciation (Thorpe et al. 2010, Muñoz et al. 2013). We found that environmental variation induces shifts in the position of populations in morphospace and in the volume of morphospace occupied. However, natural selection does not act alone in generating intraspecific morphological diversity across environments; sexual selection may play a role, alongside nonadaptive responses that primarily affect sexually selected traits. Nonetheless, without direct measures of sexual selection in the field, these remain untested hypotheses. Our results also demonstrate that the strength of trait clustering, along with spatial trait-environment relationships, can differ between sexes. Moving forward, explicitly considering sex differences in such analyses will provide new insights into the processes influencing ecological radiation (Butler et al. 2007, Pincheira-Donoso et al. 2009). Lastly, studies that have evaluated trait clustering or evenness to infer assembly processes using just a single sex may have missed key aspects of the structure of trait variation and the processes driving it.

\section{ACKNOWLEDGEMENTS}

Thanks to Jorge Lobo for sharing the Tenerife climate and elevation data. Leslie Bode provided assistance in the field and Yoel Stuart provided useful advice. James Hicks and the rest of the University of Nottingham's BEER (biogeography, ecology and evolution research) group provided valuable feedback on earlier drafts. MLD was supported by the EU Seventh Framework Programme (FP7/2007-2013) in the form of a Marie Curie International Outgoing Fellowship.

\section{REFERENCES}

Abouheif, E. and Fairbairn, D. 1997. A comparative analysis of allometry for sexual size dimorphism: assessing Rensch's rule. - Am. Nat. 149: 540-562.

Algar, A. C. and Mahler, D. L. 2015. Area. climate heterogeneity, and the response of climate niches to ecological opportunity in island radiations of Anolis lizards. - Global Ecol. Biogeogr. DOI: 10.1111/geb.12327. 
Algar, A. C. et al. 2011. Quantifying the importance of regional and local filters for community trait structure in tropical and temperate zones. - Ecology 92: 903-914.

Angilletta, M. J. et al. 2004. Temperature, growth rate, and body size in ectotherms: fitting pieces of a life-history puzzle. - Integr. Comp. Biol. 44: 498-509.

Arechavaleta, M. et al. (eds) 2009. Lista de especies silvestres de Canarias. Hongos, plantas y animales terrestres. - Gobierno de Canarias.

Arnedo, M. A. et al. 2001. Radiation of the spider genus Dysdera (Araneae, Dysderidae) in the Canary Islands: cladistic assessment based on multiple data sets. - Cladistics 17: 313-353.

Ashton, K. G. and Feldman, C. R. 2003. Bergmann's rule in nonavian reptiles: turtles follow it, lizards and snakes reverse it. - Evolution 57: 1151-1163.

Bickel, R. and Losos, J. B. 2002. Patterns of morphological variation and correlates of habitat use in Chameleons. - Biol. J. Linn. Soc. 76: 91-103.

Blanckenhorn, W. U. et al. 2006. When Rensch meets Bergmann: does sexual size dimorphism change systematically with latitude? - Evolution 60: 2004-2011.

Blankers, T. et al. 2012. Ecological radiation with limited morphological diversification in salamanders. - J. Evol. Biol. 25: 634-646.

Blonder, B. 2015. hypervolume: high-dimensional kernel density estimation and geometry operations. R package version 1.3.0. http://cran.r-project.org/package=hypervolume.

Blonder, B. et al. 2014. The n-dimensional hypervolume. - Glob. Ecol. Biogeogr. 23: 595-609.

Bolnick, D. I. and Doebeli, M. 2003. Sexual dimorphism and adaptive speciation: two sides of the same ecological coin. - Evolution 57: 2433-2449.

Bolnick, D. I. et al. 2011. Why intraspecific trait variation matters in community ecology. - Trends Ecol. Evol. 26: 183-192.

Butler, M. A. and Losos, J. B. 2002. Multivariate sexual dimorphism, sexual selection, and adaptation in Greater Antillean Anolis lizards. - Ecol. Monogr. 72: 541-559.

Butler, M. A. et al. 2000. The relationship between sexual size dimorphism and habitat use in Greater Antillean Anolis lizards. - Evolution 54: 259-272. 
Butler, M. A. et al. 2007. Sexual dimorphism and adaptive radiation in Anolis lizards. - Nature 447: 202-205.

Cornwell, W. K. et al. 2006. A trait-based test for habitat filtering: convex hull volume. - Ecology 87: $1465-1471$.

Cox, R. M. and Calsbeek, R. 2010. Sex-specific selection and intraspecific variation in sexual size dimorphism. - Evolution 64: 798-809.

Cox, R. M. et al. 2003. A comparative test of adaptive hypotheses for sexual size dimorphism in lizards. - Evolution 57: 1653-1669.

Cox, S. C. et al. 2010. Divergence times and colonization of the Canary Islands by Gallotia lizards. Mol. Phylogenet. Evol. 56: 747-757.

Fairbairn, D. J. et al. (eds) 2007. Sex, size and gender Roles: evolutionary studies of sexual dimorphism. - Oxford University Press.

Fernández-Palacios, J. M. and Nicolás, J. P. 1995. Altitudinal pattern of vegetation variation on Tenerife. - J. Veg. Sci. 6: 183-190.

Fernández-Palacios, J. M. and Whittaker, R. J. 2008. The Canaries: an important biogeographical meeting place. - J. Biogeogr. 35: 379-387.

Fitch, H. S. 1981. Sexual size differences in reptiles. - Kansas Museum of Natural History.

Forsman, A. and Shine, R. 1995. The adaptive significance of colour pattern polymorphism in the Australian scincid lizard Lampropholis delicata. - Biol. J. Linn. Soc. 55: 273-291.

Herrel, A. et al. 1999. Sexual dimorphism of head size in Gallotia galloti: testing the niche divergence hypothesis by functional analyses. - Funct. Ecol. 13: 289-297.

Hortal, J. and Lobo, J. M. 2005. An ED-based protocol for optimal sampling of biodiversity. Biodivers. Conserv. 14: 2913-2947.

Hortal, J. et al. 2007. Limitations of biodiversity databases: case study on seed-plant diversity in Tenerife, Canary Islands. - Conserv. Biol. 21: 853-863.

Hudson, E. J. and Price, T. D. 2014. Pervasive reinforcement and the role of sexual selection in biological speciation. - J. Hered. 105 Suppl: 821-833. 
Huyghe, K. et al. 2005. Morphology, performance and fighting capacity in male lizards, Gallotia galloti. - Funct. Ecol. 19: 800-807.

Huyghe, K. et al. 2007. Morphology, performance, behavior and ecology of three color morphs in males of the lizard Podarcis melisellensis. - Integr. Comp. Biol. 47: 211-220.

Irschick, D. J. et al. 2008. How does selection operate on whole-organism functional performance capacities? A review and synthesis. - Evol. Ecol. Res. 10: 177-196.

Johnson, M. A. et al. 2010. Behavioral convergence and adaptive radiation: effects of habitat use on territorial behavior in Anolis lizards. - Evolution. 64: 1151-1159.

Kaliontzopoulou, A. et al. 2010. Intraspecific ecomorphological variation: linear and geometric morphometrics reveal habitat-related patterns within Podarcis bocagei wall lizards. - J. Evol. Biol. 23: 1234-1244.

Kaliontzopoulou, A. et al. 2014. Ecomorphological variation in male and female wall lizards and the macroevolution of sexual dimorphism in relation to habitat use. - J. Evol. Biol. 28: 80-94.

Laiolo, P. et al. 2013. Local climate determines intra- and interspecific variation in sexual size dimorphism in mountain grasshopper communities. - J. Evol. Biol. 26: 2171-2183.

Leal, M. and Fleishman, L. J. 2002. Evidence for habitat partitioning based on adaptation to environmental light in a pair of sympatric lizard species. - Proc. R. Soc. B - Biol. Sci. 269: 351359.

Lopez-Darias, M. et al. 2014. Sex-specific differences in ecomorphological relationships in lizards of the genus Gallotia. - Funct. Ecol. 29: 506-514.

Losos, J. B. and Schluter, D. 2000. Analysis of an evolutionary species-area relationship. - Nature 408: 847-850.

Losos, J. B. and Mahler, D. L. 2010. Adaptive radiation: the interaction of ecological opportunity, adaptation, and speciation. - In: Bell, M. A. et al. (eds), Evolution since Darwin: The First 150 Years. Sinauer Associates, pp. 381-420.

Losos, J. B. et al. 2000. Evolutionary implications of phenotypic plasticity in the hindlimb of the lizard Anolis sagrei. - Evolution (N. Y). 54: 301-305. 
Mahler, D. L. et al. 2013. Exceptional convergence on the macroevolutionary landscape in island lizard radiations. - Science 341: 292-295.

Measey, G. J. et al. 2009. Morphology, ornaments and performance in two chameleon ecomorphs: is the casque bigger than the bite? - Zoology 112: 217-226.

Melville, J. and Swain, R. 2000. Evolutionary relationships between morphology, performance and habitat openness in the lizard genus Niveoscincus (Scincidae: Lygosominae). - Biol. J. Linn. Soc. 70: $667-683$.

Molina-Borja, M. 2003. Sexual dimorphism of Gallotia atlantica atlantica and Gallotia atlantica mahoratae (Lacertidae) from the Eastern Canary Islands. - J. Herpetol. 37: 769-772.

Molina-Borja, M. et al. 1997. Intrapopulation variability in morphology, coloration, and body size in two races of the lacertid lizard, Gallotia galloti. - J. Herpetol. 31: 499-507.

Molina-Borja, M. et al. 2006. Sex and population variation in ultraviolet reflectance of colour patches in Gallotia galloti (Fam. Lacertidae) from Tenerife (Canary Islands). - J. Zool. 268: 193-206.

Molina-Borja, M. et al. 2010. Sexual size and shape dimorphism variation in Caesar's lizard (Gallotia caesaris, Lacertidae) from different habitats. - J. Herpetol. 44: 1-12.

Muñoz, M. M. and Hewlett, J. 2011. Ecological consequences of continual volcanic activity on the lizard, Anolis lividus, from Montserrat. - Herpetol. Rev. 42: 160-165.

Muñoz, M. M. et al. 2013. Divergence in coloration and ecological speciation in the Anolis marmoratus species complex. - Mol. Ecol. 22: 2668-2682.

Muñoz, M. M. et al. 2014a. Untangling intra- and interspecific effects on body size clines reveals divergent processes structuring convergent patterns in Anolis lizards. - Am. Nat. 184: 636-646.

Muñoz, M. M. et al. 2014b. Evolutionary stasis and lability in thermal physiology in a group of tropical lizards. - Proc. R. Soc. B - Biol. Sci. 281: 20132433.

Ord, T. et al. 2010. Adaptation and plasticity of animal communication in fluctuating environments. Evolution 64: 3134-3148.

Panhuis, T. M. et al. 2001. Sexual selection and speciation. - Trends Ecol. Evol. 16: 364-371.

Pincheira-Donoso, D. and Meiri, S. 2013. An intercontinental analysis of climate-driven body size clines in reptiles: no support for patterns, no signals of processes. - Evol. Biol. 40: 562-578. 
Pincheira-Donoso, D. et al. 2008. The evolution of body size under environmental gradients in ectotherms: why should Bergmann's rule apply to lizards? - BMC Evol. Biol. 8: 68.

Pincheira-Donoso, D. et al. 2009. A phylogenetic analysis of sex-specific evolution of ecological morphology in Liolaemus lizards. - Ecol. Res. 24: 1223-1231.

Pinheiro J. et al. 2014. nlme: linear and nonlinear mixed effects models. R package version 3.1-118.

Pinto, G. et al. 2008. Testing the island effect in adaptive radiation: rates and patterns of morphological diversification in Caribbean and mainland Anolis lizards. - Proc. R. Soc. B Biol. Sci. 275: 2749-2757.

R Core Team 2015. R: A language and environment for statistical computing. Version 3.2.1 - R Foundation for Statistical Computing. http://www.R-projet.org/.

Ricklefs, R. E. and Lovette, I. J. 1999. The roles of island area per se and habitat diversity in the species-area relationships of four Lesser Antillean faunal groups. - J. Anim. Ecol. 68: 11421160.

Ritchie, M. G. 2007. Sexual selection and speciation. - Annu. Rev. Ecol. Evol. Syst. 38: 79-102.

Ruxton, G. D. and Neuhäuser, M. 2013. Improving the reporting of P-values generated by randomization methods. - Methods Ecol. Evol. 4: 1033-1036.

Schluter, D. 2000. The ecology of adaptive radiation. - Oxford University Press.

Schoener, T. W. 1967. The ecological significance of sexual dimorphism in size in the lizard Anolis conspersus. - Science (80-. ). 155: 474-477.

Sears, M. W. and Angilletta, M. J. 2004. Body size clines in Sceloporus lizards: proximate mechanisms and demographic constraints. - Integr. Comp. Biol. 44: 433-42.

Shine, R. 1989. Ecological causes for the evolution of sexual dimorphism: a review of the evidence. Q. Rev. Biol. 64: 419-461.

Stillwell, R. C. and Fox, C. W. 2009. Geographic variation in body size, sexual size dimorphism and fitness components of a seed beetle: local adaptation versus phenotypic plasticity. - Oikos 118: $703-712$.

Swenson, N. G. et al. 2012. The biogeography and filtering of woody plant functional diversity in North and South America. - Glob. Ecol. Biogeogr. 21: 798-808. 
Thorpe, R. S. and Baez, M. 1987. Geographic variation within an island: univariate and multivariate contouring of scalation, size, and shape of the lizard Gallotia galloti. - Evolution 41: 256-268.

Thorpe, R. S. et al. 1996. Matrix correspondence tests on the DNA phylogeny of the Tenerife Lacertid elucidate both historical causes and morphological adaptation. - Syst. Biol. 45: 335-343.

Thorpe, R. S. et al. 2010. Genetic tests for ecological and allopatric speciation in anoles on an island archipelago. - PLoS Genet. 6: e1000929.

Villéger, S. et al. 2008. New multidimensional functional diversity indices for a multifaceted framework in functional ecology. - Ecology 89: 2290-2301.

Villéger, S. et al. 2011. The multidimensionality of the niche reveals functional diversity changes in benthic marine biotas across geological time. - Ecol. Lett. 14: 561-568.

Violle, C. et al. 2012. The return of the variance: intraspecific variability in community ecology. Trends Ecol. Evol. 27: 244-252.

Vitales, D. et al. 2014. The explosive radiation of Cheirolophus (Asteraceae, Cardueae) in Macaronesia. - BMC Evol. Biol. 14: 118.

Weiher, E. and Keddy, P. A. 1995. Assembly rules, null models, and trait dispersion: new questions from old patterns. - Oikos 74: 159-164.

Weiher, E. et al. 1998. Community assembly rules, morphological dispersion, and the coexistence of plant species. - Oikos 81: 309-322.

Zobel, M. et al. 2011. The formation of species pools: historical habitat abundance affects current local diversity. - Glob. Ecol. Biogeogr. 20: 251-259. 
Table 1. Eigenvectors for the first three principal

components from a principal components analysis on lizard

morphology after varimax rotation. Right legs were

measured and toe measurements were taken on the fourth toe on the right side. Head traits are shown in Fig. A3.

\begin{tabular}{lccc}
\hline Variable (residuals) & PC1 & PC2 & PC3 \\
\hline femur & -0.043 & -0.079 & 0.428 \\
tibia & -0.099 & -0.312 & 0.263 \\
hindtoe & -0.028 & -0.567 & 0.070 \\
humerus & 0.071 & 0.051 & 0.618 \\
ulna & -0.020 & -0.085 & 0.488 \\
foretoe & -0.053 & -0.552 & -0.036 \\
head length & -0.401 & -0.074 & -0.061 \\
pileus width & -0.349 & 0.304 & 0.131 \\
head height & -0.362 & 0.322 & 0.114 \\
head width & -0.294 & 0.124 & 0.195 \\
snout length & -0.410 & -0.127 & -0.200 \\
lower jaw I & -0.401 & -0.139 & -0.088 \\
lower jaw II & -0.387 & -0.094 & -0.033
\end{tabular}

621 
Table 2. Wald $F$-test results for terms in linear mixed effects models evaluating morphological differences between Gallotia galloti in different environments and between sexes on Tenerife. When the ENV $\times$ SEX interaction was not significant $(\alpha=0.05)$, a reduced model was fitted. All tests are based on marginal sums of squares. The models for head size incorporated unequal variances among environment because $\mathrm{AIC}_{\mathrm{c}}$ indicated this provided better fit than assuming equal variances (this was not the case for the other traits).

\begin{tabular}{|c|c|c|c|c|c|c|c|c|c|}
\hline \multirow[b]{2}{*}{ Trait } & \multicolumn{3}{|c|}{ ENV } & \multicolumn{3}{|c|}{ SEX } & \multicolumn{3}{|c|}{$\mathrm{ENV} \times \mathrm{SEX}$} \\
\hline & $F$ & $\mathrm{df}$ & $\mathrm{P}$ & $F$ & df & $\mathrm{P}$ & $F$ & $\mathrm{df}$ & $\mathrm{P}$ \\
\hline
\end{tabular}

Full Model

$\begin{array}{lccccccccc}\log (\mathrm{SVL}) & 0.86 & 3,16 & 0.48 & 1837.75 & 1,328 & <0.01 & 7.69 & 3,328 & <0.01 \\ \text { Head Size (PC1) } & 4.37 & 3,16 & 0.020 & 26.81 & 1,328 & <0.01 & 1.21 & 3,328 & 0.31 \\ \text { Toe Length (PC2) } & 1.32 & 3,16 & 0.30 & 1.83 & 1,328 & 0.18 & 0.43 & 3,328 & 0.73 \\ \text { Leg Length (PC3) } & 4.78 & 3,16 & 0.015 & 14.23 & 1,328 & <0.01 & 0.13 & 3,328 & 0.94\end{array}$

Reduced Model

$\log (\mathrm{SVL})$

Head Size (PC1)

$\begin{array}{lll}4.33 & 3,16 & 0.021\end{array}$

$26.78 \quad 3,331$

$<0.01$

Toe Length (PC2)

$\begin{array}{lll}1.34 & 3,16 & 0.29\end{array}$

$1.84 \quad 1,331$

0.18

Leg Length (PC3)

$\begin{array}{lll}4.74 & 3,16 & 0.015\end{array}$

$14.35 \quad 1,331$

$<0.01$ 


\section{FIGURE LEGENDS}

Figure 1. Male (upper) and female (lower) Gallotia galloti on Tenerife. Images are scaled so that head length approximately matches the mean difference among males and females.

Figure 2. Four environmental zones on Tenerife generated using $k$-means clustering on climate, elevation and aspect at $500 \mathrm{~m}$ resolution, accounting for $70 \%$ of the environmental variation on the island. Blue diamonds indicate sampling localities.

Figure 3. Male and female traits in four different environments on Tenerife. Head size, toe length and leg length are all corrected for body size (SVL). Environment letters correspond to the map in Figure 2. Principal component loadings are given in Table 2.

Figure 4. Morphospace occupied by male and female Gallotia galloti on Tenerife. Convex hulls were drawn using twenty randomly chosen males and twenty randomly chosen females. SVL is log snoutvent length, -HS is head size (negatively scaled so that small values equal small heads), and LL is leg length. All axes were standardized to zero mean and unit variance.

Figure 5. The position and volume of morphospace occupied by populations of Gallotia galloti in different environments on Tenerife. Column one shows the convex hulls of populations from the four environments (A - D in Figure 3), column two shows the convex hull volume for lizards from each environment, and column three depicts the distance of the population's morphospace centroid from the centroid when all populations were pooled. Rows show results for both sexes pooled, and males and females only. Values are based on 20 randomly chosen males and females from each environment. ** indicates a P-value less than 0.05 based on our null model. * indicates a P-value with a $95 \%$ confidence interval that spans 0.05 . SVL is log snout-vent length, -HS is head size (negatively scaled so that small values equal small heads), and LL is leg length. All axes were standardized to zero mean and unit variance. 
661 Figure 1

662



663

664

665

666 
667 Figure 2

668

669

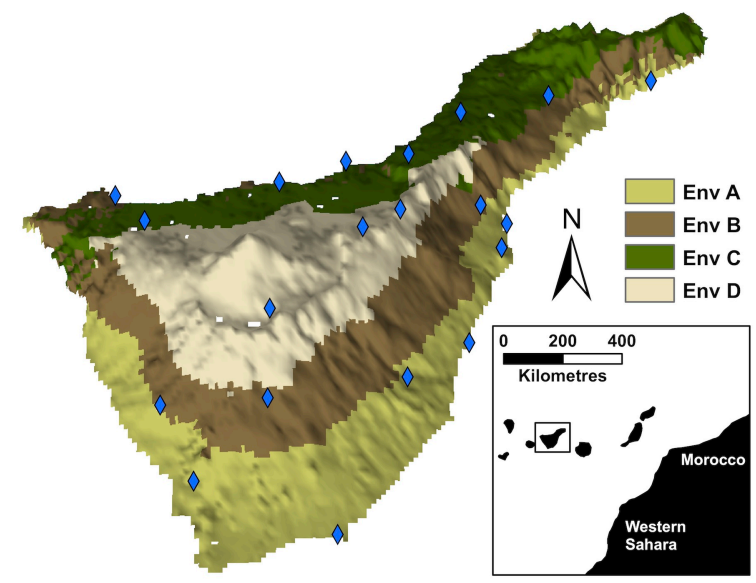

670

671

672 
$673 \quad$ Figure 3

674
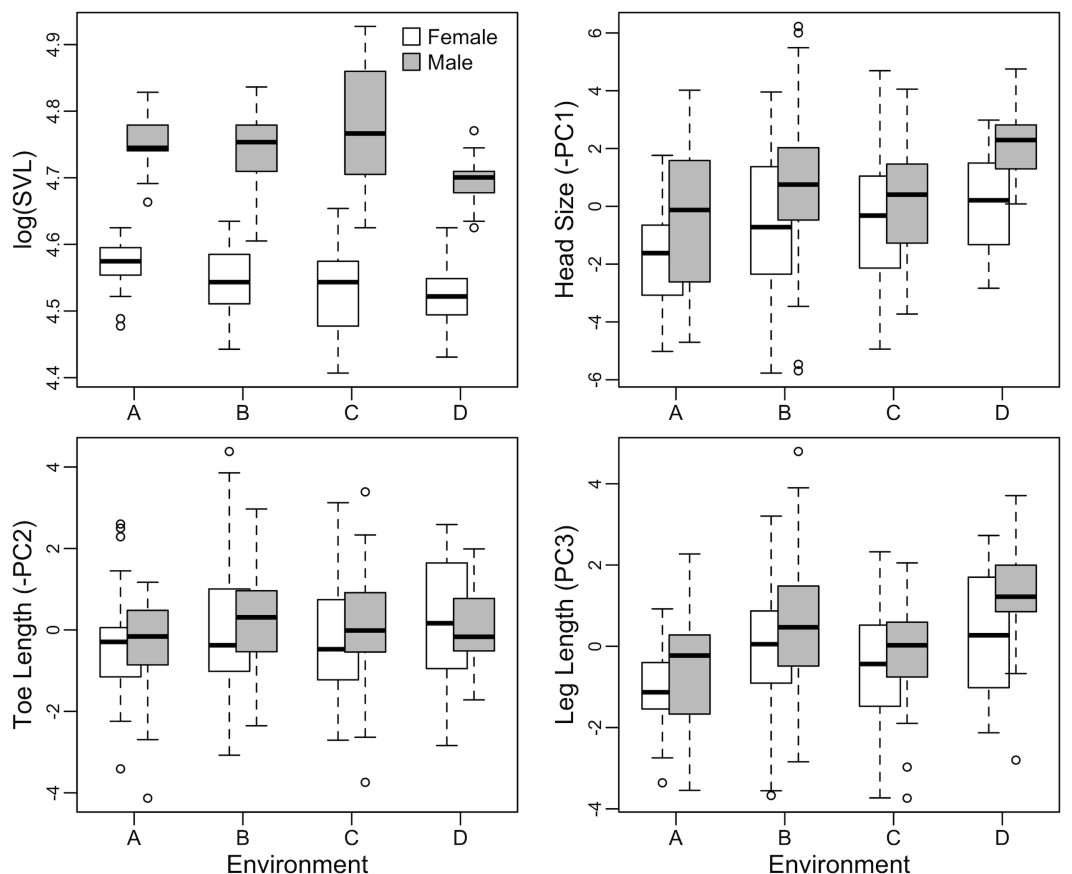

675

676

677

678 
$679 \quad$ Figure 4

680

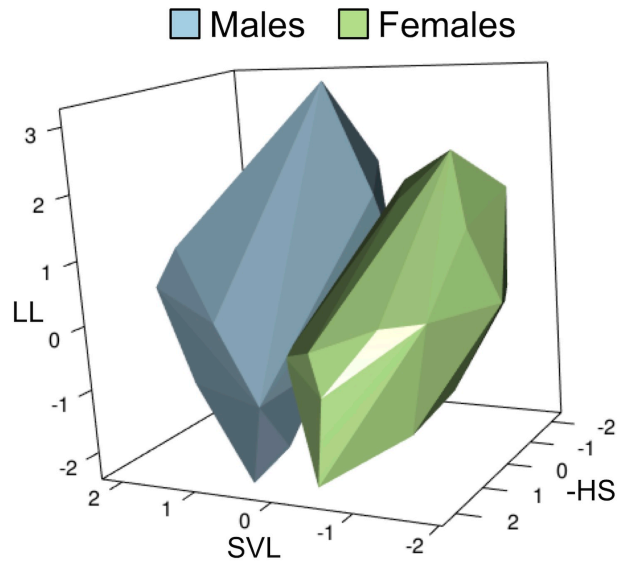

681

682

683

684 
Figure 5

686
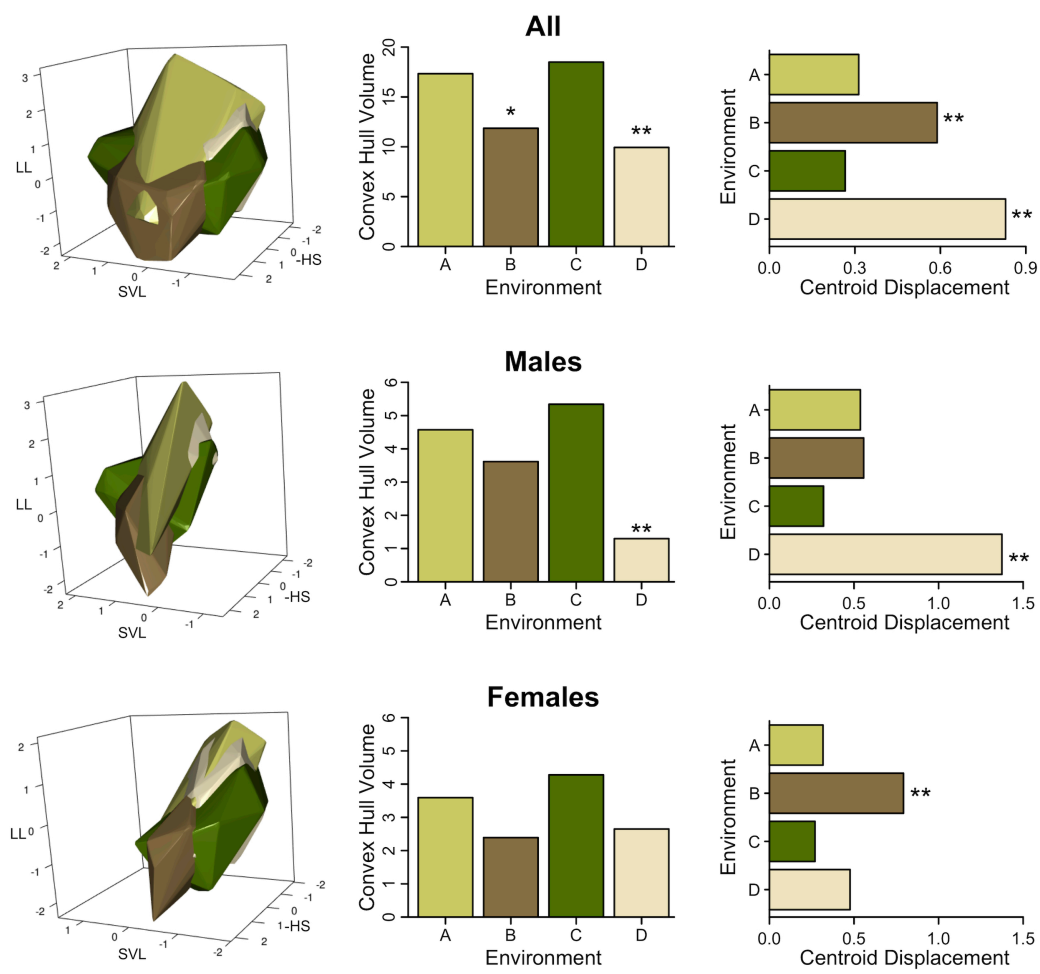